\title{
Membranes as the Mediators of Flow of Matter, Energy and Information
}

\author{
Arnošt KoTYK \\ Institute of Physiology, Czechoslovak Academy of Sciences, \\ Videñská 1083, 14220 Prague 4, Czechoslovakia
}

(Received November 26, 1990)

\begin{abstract}
Bilayer lipid structures, generically called (bio)membranes, constitute the principal construction blocks of all cells. Their primordial function apparently was to separate compartments of different composition and this is now achieved by a variety of transport systems positioned across the membranes. These systems catalyze the flow of substances dissolved in the surrounding aqueous media into and out of cells and organelles. Early in evolution some membranes acquired the ability to mediate interconversion of different kinds of energy (chemical, osmotic, electrical, mechanical). Later in evolution many membranes were endowed with the ability to receive physical and chemical signals and transduce the information contained in them into meaningful intracellular regulatory events. All the three categories of membrane functions require movement of membrane components both along the membrane plane and, intramolecularly, across the membrane. Although virtually all the specific functions are mediated by proteins the surroudning lipids are now recognized to play a significant role in maintaining and regulating the protein functions and, in some cases, specifically perform receptor functions.
\end{abstract}

KEY WORDS: Membrane Transport / Energy Conversion / Signal Transduction / Nonspecific Permeation / Specific Transport / Group Translocation / Active transport / Electrochemical Potential / G Proteins / Neurotransmitters

In the biological realm on this planet virtually all life is associated with the existence of cells. In spite of the tremendous variety of cell sizes and forms - they range from $10^{-6} \mathrm{~m}$ to $10^{1} \mathrm{~m}$ in length and from simple spheres to corrugated blobs full of protuberances or funnel-shaped bodies on a stalk, all cells are built from only a few fundamental structural components.

Al1 cells are constructed from lipid (plus protein) membranes, all cells use nucleic acid (plus protein) chromosomes and ribosomes for reproduction, all eukaryotic (nucleus-bearing) cells are reinforced from within by a protein cytoskeleton and all bacterial, fungal and plant cells (with very few exceptions) are surrounded by a more or less rigid carbohydrate (plus protein) cell wall. These four structural - functional principles obviously play roles of different importance in the individual cell development (ontogeny) as well as in its evolutionary trend (phylogeny) but, at least for a limited period of time, a cell will sur- 
vive without chromosomes, without the cytoskeleton and without the cell wall but it cannot exist without membranes.

Membranes and their functions are thus understandably in the focus of attention of cell biologists, as well as of modern biochemists and biophysicists. With the exception of the genetics of cells and organisms, membranes are involved in all major processes characterizing living systems, viz. uptake of nutrients and their metabolism to provide both building materials and energy, absorption of energy to ensure the continuation of cell's life, interaction with the surroundings in terms of receiving various signals and transforming them into others, as well as adapting the cell's behavior to minimize adverse environmental influences.

Using a systems theory approach we can describe the function of membranes as mediation of flow of matter, flow of energy and flow of information. Let us now deal with these categories one by one.

\section{FLOW OF MATTER}

Some aspects of movement of molecules in membranes are trivial in the present context, e.g. the lateral diffusion of membrane components in the membrane plane or fluctuations in molecular orientation caused by thermal agitation. We shall rather address reactions where extraneous molecules or ions take part.

\subsection{Enzyme reactions in membranes}

It can be estimated that of the approximately 2000 enzymes now known, perhaps 200 are anchored in cell membranes and further 800 are loosely associated with membranes. The membrane connection has obviously two advantages:

a. If the enzyme is part of a reaction sequence, such as those located in inner mitochondrial or in thylakoid membranes, or those involved in steroid synthesis in the smooth endoplasmic reticulum, it is of obvious advantage if the product of one reaction serving as substrate for the next finds the appropriate enzyme by two- rather than three-dimensional diffusion. In some cases the enzymes may be so organized that the transit time for the intermediate is virtually zero.

b. Attachment to membranes provides greater thermal stability for the enzyme. This is apparently a necessity with extremely thermophilic organisms, such as many archaebacteria (Thermoplasma, Thermoproteus) and bacteria (Thermus, Thermoactinomyces). Whereas the growth and metabolic optima of some of these may be near $100^{\circ} \mathrm{C}$, the temperature optima of their isolated enzymes lie at $40-50^{\circ} \mathrm{C}$.

\subsection{Transport across membranes}

Substances dissolved or dispersed in the aqueous medium adjacent to a membrane can cross it in three principally different ways: (a) by passive, nonspecific permeation through the membrane, (b) by a specific, protein-involving enzyme-like reaction, (c) by a process altering transiently the membrane structure.

\subsubsection{Nonspecific permeation}

Processes of nonspecific permeation are characterized by simple kinetics, obeying Fick's First Law of diffusion, modified for one co- 
ordinate axis and taking into account the membrane electrical potential, thus

$$
J_{\mathrm{s}}=-(D / 1)\left(c_{\mathrm{s}}^{\prime} \mathrm{e}^{-n F \Delta \psi / 2 R T}-c_{\mathrm{s}}^{\prime \prime} \mathrm{e}^{n F \Delta \psi / 2 R T}\right)=P\left(c_{\mathrm{s}}^{\prime} \zeta^{1 / 2}-c_{\mathrm{s}}^{\prime \prime} \zeta^{-1 / 2}\right)
$$

where $J_{S}$ is the flux across the membrane, $D$ is the intramembrane diffusion coefficient, I the membrane thickness, $C_{s}^{\prime}$ and $c_{s}^{\prime \prime}$ the solute concentrations at the starting and at the target side, respectively, $n$ is the number of positive charges on the transported solute, $F$ then the Faraday constant, $\Delta \psi$ the electrical potential difference across the membrane (taken as a negative number), $R$ is the gas constant, $T$ then the absolute temperature, $P$ is the permeability constant $(D / I)$ and $\zeta=\exp (-n F \Delta \psi / R T)$.

Clearly, in transport equilibrium when $J_{s}=0$ it must be true that the ratio $c_{s}^{\prime \prime} / c_{s}^{\prime}$ is equal to unity for uncharged solutes and to $\zeta$ for ions.

The initial rate, when $c_{\mathrm{s}}^{\prime \prime}=0$, is linearly proportional to concentration; this is why these systems are sometimes called 'simple diffusion'.

Several possibilities offer themselves to a molecule or ion for crossing a membrane.

a. Those that are fairly soluble in lipids distribute themselves between the membrane interior and both aqueous phases according to their partition coefficient. The rate of this process is virtually unaffected by the physical state of the membrane lipids.

b. Membrane crossing by any dissolved molecule or ion is facilitated above the temperature of phase transition (from $L_{\beta}$ or rather $P_{\beta}$ phase to $L_{\alpha}$ ) by the formation of kinks in the lipid structure, caused by transgauche changes in alkyl or alkenyl chain configuration.

c. Membrane proteins or their complexes frequently offer avenues for passive membrane transport of various solutes. However, the pores within or adjacent to such proteins being rather narrow (in most eukaryotic cell membranes the effective radius is $0.2-0.4 \mathrm{~nm}$ ), the individual molecules or ions can move through such a pore only in a single file which gives rise to more complicated, in fact saturation, kinetics.

\subsubsection{Specific transport}

This is by far the most common and most important group of transport mechanisms. They invariably involve a membrane-bound protein to which the transported substance transiently binds. They range from simple selective channels which could easily fall into the group of nonspecific permeation (e.g. the outer chloroplast membrane porin which permits passage of molecules with $M_{\mathrm{r}}$ up to 10 000) to extremely specific carrier proteins (e.g., the LYP system of yeast which transports exclusively lysine).

There are several ways of classifying the transport systems in this group. While a classification based on sequence homology of the transport proteins is emerging, for the time being it is more useful to proceed from kinetic and thermodynamic considerations.

\section{a. Channel-type systems}

These are characterized by the presence of a specific binding site that is accessible simultaneously from both sides of the membrane. The rate of transport through such a channel is given by

$$
J_{s}=\frac{A\left(c_{s}^{\prime} \zeta^{1 / 2}-C_{s}^{\prime \prime} \zeta^{-1 / 2}\right)}{B+C \zeta^{1 / 2} C_{s}^{\prime}+D \zeta^{-1 / 2} c_{s}^{\prime \prime}}
$$


where $A, B, C$ and $D$ are combinations of rate constants in the operation cycle of the channel. It will be seen that for the initial rate when $c_{S}^{\prime \prime}=0$ the expression turns into one analogous to the Michaelis-Menten kinetics, a segment of a rectangular hyperbola. It follows from the finite number of binding sites involved in such transport that a maximum rate is reached at high solute concentrations and this is why these and the following systems are sometimes said to display 'saturation kinetics'. However, the accumulation ratio displayed by a selective channel is the same as in nonspecific permeation, viz. $c_{\mathrm{s}}^{\prime \prime} / c_{\mathrm{s}}^{\prime}=1$ for nonelectrolytes and $c_{\mathrm{s}}^{\prime \prime} / c_{\mathrm{s}}^{\prime}=\zeta$ for ions.

Several types of such channels are now known.

aa. Nongated channels, such as the porins of outer mitochondrial, chloroplast and bacterial membranes. They show a graded selectivity for cations, occasionally anions and, infrequently, for nonelectrolyte molecules.

ab. Ligand-gated channels, such as those associated with nerve impulse transmission. These channels open upon binding an appropriate ligand. A typical example is the nicotinic acetylcholine receptor of postsynaptic membranes which opens a cation-permeable channel upon binding acetylcholine.

ac. Voltage-gated channels are the typical nerve channels, such as those for $\mathrm{Na}^{+}$but also for $\mathrm{K}^{+}$and for $\mathrm{Ca}^{2+}$. Here the spreading action potential causes the opening of a gate (reflected in a gating current) and thus permits the given ion to approach the selectivity site and from there to pass through the channel.

ad. Stretch-activated channels have been discovered only recently. They respond to mechanical tension in membranes ranging from yeast to blood capillary lining. A special case here are the stereocilia of an inner ear hair cell which, upon being displaced by as little as 0.05 $\mathrm{nm}$ due to incoming sound, tug at a cation channel gate and open it.

\section{b. Carrier-type systems}

The term 'carrier' is to be understood at the molecular level as a membrane-spanning protein with a binding site somewhere near the membrane center such that it is exposed by intrinsic motion of a molecular segment or by swinging of a nearby 'gate' either to the one or the other side of the membrane.

In common with the channels, there is a specific binding site involved but with an alternate exposure which gives rise to the following expression for the transport rate (in the simplest model considered for a steady-state system)

$$
J=\frac{A C_{\mathrm{s}}^{\prime} \zeta^{1 / 2}-A^{\prime} C_{\mathrm{s}}^{\prime \prime} \zeta^{-1 / 2}}{B+C C_{\mathrm{s}}^{\prime}+D C_{\mathrm{s}}^{\prime \prime}+E C_{\mathrm{s}}^{\prime} C_{\mathrm{s}}^{\prime \prime}}
$$

where $B, C, D$ and $E$ are more or less complicated functions of the potential. Here, like in the channel-type transport, the initial rate obeys Michaelis-Menten kinetics but, in addition, displays phenomena such as countertransport and trans-acceleration.

Moreover, the accumulation ratio $C_{\mathrm{S}}^{\prime} / C_{\mathrm{S}}^{\prime \prime}$ will be given by $A / A^{\prime}$ for nonelectrolytes and by $A \zeta^{\prime} A^{\prime}$ for ions; hence, with an input of metabolic energy into the system, uphill transport can be achieved.

Many types of carrier systems are known.

ba. Mediated or facilitated diffusion, in which no energy is required to accomplish transport - this is the case with monosaccharides in some yeasts and in a variety of vertebrate organs. 
bb. Active transport. Although basically identical with (ba) as to their transmembrane disposition and alternate exposure to the two membrane sides, active transport systems are functionally asymmetric, displaying different affinities and/or residence times at each of the membrane faces. This stems from a coupling with an energy source and results in an unequal distribution of the transported solute in pseudo-equilibrium, even if the solute is uncharged. Depending on whether the energy coupling is direct, say, with a chemical reaction, or indirect, such as through dissipation of an electrochemical potential gradient, we distinguish between primary and secondary active systems.

bba. Primary systems. The source of energy can be one of a number of different types.

(i) Photochemical reaction. Absorption of a photon with a wavelength of $568 \mathrm{~nm}$ by bacteriorhodopsin or of $578 \mathrm{~nm}$ by halorhodopsin in various strains of extremely halophilic archaebacteria causes an isomerization of a lysine-bound retinal and, owing to the ensuing change of accessibility for a proton or for a chloride anion, respectively, an active transport of $\mathrm{H}^{+}$out or of $\mathrm{Cl}^{-}$into the cell results.

(ii) Hydrolysis of the terminal phosphate group of ATP. Nearly a dozen membrane-bound adenosinetriphosphatases are knwon which trans1ocate cations $\left(\mathrm{H}^{+}\right.$or $\mathrm{H}_{3} \mathrm{O}^{+}, \mathrm{Na}^{+}, \mathrm{K}^{+}, \mathrm{Ca}^{2+}$ in various stoichiometries) as well as chloride and possibly hydrogen carbonate anions. In the P-type a phosphorylated intermediate of the protein is formed directly from ATP, in the $\mathrm{F}$ - and V-types it is not. Quite recently, ATP splitting was also found to be involved in the extrusion of xenobiotics from various eukaryotic cells by the so-called P-glycoprotein and, in bacteria, in the uptake of various amino acids by the so-called binding-protein systems.

(iii) Free energy of oxidation is used in inner mitochondrial membranes, thylakoids and some bacteria to displace protons and, exceptionally (e.g., in Vibrio alginolyticus) $\mathrm{Na}^{+}$ions against their electrochemical potential differences.

(iv) Many bacteria, particularly the alkaliphilic ones, such as Klebsiella aerogenes, Clostridium symbiosum, Propiogenum modestum, contain decarboxylation systems (e.g., oxaloacetate to pyruvate), the free energy of which is used for extruding $2 \mathrm{Na}^{+}$ions per oxo acid molecule.

$\mathrm{bbb}$. Secondary systems. Several hundred instances have been described where either $\mathrm{H}^{+}$or $\mathrm{Na}^{+}$ions bind to a carrier protein together with the substrate to be transported and, by virtue of their concentration difference as well as the membrane potential, drive the substrate transport uphill. The energy here stems from the electrochemical potential difference across the membrane

$$
\Delta \tilde{\mu}_{\text {ion }}=F \Delta \psi-R T \ln \left(c_{\text {ion }}^{\prime} / c_{\text {ion }}^{\prime \prime}\right)
$$

or, often expressed for $\mathrm{H}^{+}$as protonmotive force,

$$
\mathrm{PMF}=\Delta \psi-2.3 R T \Delta \mathrm{pH} / F
$$

A great many of the $\mathrm{H}^{+}$-binding proteins involved have been isolated and sequenced and it is quite clear that they contain a single polypeptide chain, even if it traverses the membrane as many as 14 times.

\section{c. Group translocation}

The operation of all the above saturable systems could be described by enzyme kinetics, most simply of the iso-uni-uni type, i.e. the product of the transport reaction was identical with its substrate but 
was separated from it by a membrane. There are, however, systems where the transport is effected by a true vectorially located enzyme reaction such that the substrate, say, D-glucose, is taken up at one membrane side to emerge as a different 'product', say, D-glucose-6-phosphate, at the other side. Bacterial phosphotransferases are a typical example.

\subsubsection{Transport involving restructuring of membrane}

It is clear from steric considerations that large molecules, such as proteins and nucleic acids, cannot enter cells by any of the above mechanisms where the effective diameters of the transported solutes are no more than $1 \mathrm{~nm}$. Two stratagems are used in these cases.

a. Mechanisms where receptor or pilot proteins swing through the membrane or rotate with the attached macromolecule (e.g., of DNA).

b. Mechanisms where (i) vesicles form by invagination of the membrane in response to an external ligand (or, exceptionally, without any such stimulus), pinch off and are then degraded intracellularly together with their contents - this is endocytosis, (ii) vesicles formed intracellularly fuse with the outer membrane and release their contents outward - this is exocytosis. Many such instances have been described and it appears that the vesiculation process is inherent in all eukayrotic cells.

\section{FLOW OF ENERGY}

Although a great many reactions involving free energy shifts take place in the soluble phase of the cell and although even some conversions of 'chemical' energy into other kinds (mechanical, radiant) take place outside membranes, most of the energy is released and consumed in membranes, especially those of mitochondria, chloroplasts and bacteria. However, virtually all membranes and certainly those where active transport takes place, mediate a definite flow of one kind of energy into another. If we disregard these changes (in fact, they have all been treated in section $\mathrm{bb}$ ), the following energy transformations will be described in some detail.

\subsection{Conversion of radiant energy}

Radiation arriving from the sun is by far the most important source of energy in the biosphere - only lithotrophic bacteria living in geothermal waters would survive if the sun ceased to function. The way this radiant energy is converted into biologically more useful energy forms is called photosynthesis and is composed of two principal sets of reactions, a photochemical, membrane-bound one, and a chemical, taking place in the soluble phase.

The photochemical set is localized exclusively in membrane-bound chlorosomes (in green sulfur and nonsulfur bacteria) or, in all higher cell types, in membranous thylakoids. All those membranes contain bacteriochlorophyll of several types $(a-e)$ or chlorophyll a and $b$. The radiant energy is trapped here by various antenna pigments mainly of carotenoid type and, in the form of an exciton, moves toward a special bacteriochlorophyll or chlorophyll complex where it brings about separation of charges into an electron and $a$ 'hole'. It is this separation of charges that gives rise to a high redox potential difference, with the reductant then being able to reduce $\mathrm{NAD}$ to $\mathrm{NADH}$ or NADP to NADPH. This sequence 
of reactions not only serves the subsequent chemical (dark) synthesis of sugars (except in green bacteria) but, in all photosynthetic organisms, it gives rise to an electrochemical potential gradient of protons. Depending on the type and efficiency of the systems, an absorbed photon of suitable wavelength can cause the translocation of as many as $3 \mathrm{H}^{+}$ions.

\subsection{Conversion of free energy of oxidation}

Oxidation processes represent the principal source of available free energy to be used for a variety of purposes by the cell. The form into which this free energy of oxidation is converted is not ATP but the electrochemical potential gradient of protons and, exceptiorally, sodium ions. This is in fact the type of primary transport that was mentioned in section bba (iii). The underlying mechanism is mainly a type of membrane Bohr effect, based on sequential conformational changes (and hence different effective $\mathrm{p} K$ values of the proton-binding groups) brought about by the passage of electrons through the appropriate redox centers, most of them containing nonheme iron-sulfur configurations.

Presumably analogous systems operate with $\mathrm{Na}^{+}$ions in some bacteria.

A very minor part of oxidative free energy is converted into light. Bioluminescence is a process found in a variety of organisms, ranging from bacteria, to coelenterates to worms to insects. The observed light emission is always associated with the return of a singlet oxygen to its ground state and involves intermediary formation of a strained dioxyketanone ring. The wavelengths of the emitted light range from $460 \mathrm{~nm}$ for the Cypridina luciferin monoxygenase to $602 \mathrm{~nm}$ for the Photinus luciferin 4-monooxygenase.

\subsection{Conversion of electrochemical potential gradients of ions}

Either $\mathrm{H}^{+}$or $\mathrm{Na}^{+}$ions can be pumped by the appropriate system (ATPase or an oxidative complex) to form a gradient of their electrochemical potential. This gradient can then be used in various ways.

\subsubsection{Synthesis of high-energy phosphate bonds}

While a number of compounds containing 'high-energy' $P \sim 0$ bonds are synthsized outside membranes in the cytosol - these include adenosine triphosphate (ATP), guanosine triphosphate (GTP), phosphoenolpyruvate (PEP) or inorganic diphosphate (PP), the vast majority of ATP, perhaps $98 \%$ of the biosphere total, is synthesized in inner mitochondrial, thylakoid and bacterial membranes. The enzymes responsible for this synthesis are called ATP synthases (or F-type ATPases) and occur as highly complex assemblies with relative molar masses of up to 650.000 , where ADP and inorganic phosphate are bound at adjacent sites, ligated with a minimum input of energy, and ATP is released in a highly endergonic reaction, energized by a proton-associated conformational change of the protein. The passage of $3 \mathrm{H}^{+}$or $3 \mathrm{H}_{3} \mathrm{O}^{+}$ions through the membraneembedded channel of the ATPase is linked with the synthesis of 1 ATP molecule. Considering the efficiency of oxidation as generator of the electrochemical potential of protons this is in full stoichiometric agreement, with the maximum $\operatorname{ATP} / \frac{1}{2} \mathrm{O}_{2}$ ratio being 3 .

Like before, in some alkaliphilic bacteria there appears to function an analogous $\mathrm{Na}^{+}$-dependent ATP synthase. 


\subsubsection{Heat}

In the so-called brown-fat mitochondria (brown fat occurs in newborn and hibernating mammals) the electrochemical potential difference is converted entirely into heat, unlike in cells with normal mitochondria, where heat production only accompanies the splitting of ATP. The inner mitochondrial membrane in brown-fat cells is effectively short-circuited so that no $\Delta \tilde{\mu}_{\mathrm{H}}{ }^{+}$forms, this being effected by the so-called uncoupling protein or thermogenin, a well-characterized channel for $\mathrm{H}^{+}$(and probably also $\mathrm{Cl}^{-}$in the opposite direction).

\subsubsection{Electric energy}

A number of 'electric' fish species, such as electric eel, sting ray, electric catfish and stargazer, contain a special organ composed of several thousand stacked cells, whose membranes, in a resting state, bear the commonly found potential difference of $-90 \mathrm{mV}$. Through cholinergic innervation at one cell surface only, an action potential repolarizes the membrane to, say, $+40 \mathrm{mV}$, whereby a series of alternating potentials arises throughout the cell column. The discharge of the summed potential of $100-600 \mathrm{~V}$ then occurs by closing an external circuit, generally with the fish's prey.

\subsubsection{Mechanical energy}

A typical membrane-bound conversion of the energy stored in the electrochemical potential gradient of $\mathrm{H}^{+}$or $\mathrm{Na}^{+}$is the rotation of prokaryotic flagella. Unlike eukaryotic flagella, much larger structures where sliding of microtubules energized by dynein-catalyzed ATP splitting is involved in the rotation, bacterial flagella are rigid structures shaped like an early ship screw propeller, anchored in the membrane like a rotor in a stator. The passage of protons through the system and their interaction with suitably localized negative charges make the rotation of the flagellum possible. An estimated 256 protons are required for one turn of the flagellum.

\section{FLOW OF INFORMATION}

With 1ittle or no transport of matter and with negligible primary flow of energy, entropy can be transmitted to cells via interactions of membrane receptors with information-containing molecules or physical signals arriving from the outside world. The number of such signals is enormous and it must suffice for our purposes to give a brief review.

\subsection{Physical signals \\ 3.1.1. Light as a signal}

Unlike in the case of utilization of radiation as a source of energy, light is perceived as a signal by various cell membranes. In many microorganisms, ranging from purple bacteria, to protozoans, as well as in eyeless mites, pigments are found that absorb specific wavelengths of light and, by a poorly known mechanism, cause the cell to react either through photokinesis (movement in general), phototaxis (movement toward light) or photophobic response (sudden movement away from light).

At a higher level, in the eye retina light is absorbed by visual rhodopsin(s) and, via a cascade of reactions, involving a G-protein 
called transducin and cyclic GMP, causes $\mathrm{Na}^{+}$channels to close in vertebrates and to open in invertebrates. The amplification of the original signal by the series of reactions is such that a single photon may regulate the flow of $10^{6} \mathrm{Na}^{+}$ions.

\subsubsection{Temperature as a signal}

Although both some prokaryotic and virtually all eukaryotic organisms respond to increased or decreased temperature and although the receptors for temperature and its changes are known to reside in membranes, literally nothing is known about the reception and transduction of the temperature signal.

\subsubsection{Electric and magnetic fields as signals}

If we disregard the response of some nerve channels to an approaching electric field preceding the action potential there remain some ill-known sensitivities to electric fields in various organisms, including hypersensitive humans. Little is known about this at the molecular level.

To a degree, this resembles the sensing of magnetic fields by higher organisms, in particular migratory birds (and fish?). There is, however, one clear-cut case of sensing a magnetic field and that is the so-called magnetobacteria which contain, at the base of their flagellum, magnetite grains that orient themselves according to the dip of the geomagnetic lines of force and swim in a direction toward optimum levels of food and oxygen.

\subsubsection{Response to mechanical pressure}

Two groups of phenomena can be cited here.

a. Response of a cell to contact with a solid substratum. Several proteins are synthesized and transported to the cell surface and along its plane upon contact with mechanical support, among them fibronectin, laminin, chondronectin and enactin. The actual stimulus for this synthesis and translocation may be mediated by surface glycoproteins or even glycolipids.

b. The inner ear contains thousands of special hair cells bearing on their surface 50-100 projections called stereocilia. These projections are very close to a multicellular tectorial membrane which is activated by incoming sound. As the tectorial membrane touches some of the sterocilia they are tilted (the distance may be as little as $0.05 \mathrm{~nm})$. This is enough for a purely mechanical tug on a membrane channel gate in the cell body whereby $\mathrm{Ca}^{2+}$ ions pour into the cell and start a series of reactions, eventually leading to a nerve impulse traveling to the brain.

\subsection{Chemical signals}

Chemical substances of singular importance for a cell's survival as well as for a higher organism's well-being abound in the environment. Both microorganisms and multicellular organisms receive such signals at the outer membrane and respond to them in various ways. (Intracellular signaling also occurs, e.g., the action of steroid hormones, but this will not concern us here.) 


\subsubsection{Bacterial taxis}

Motile bacteria respond to chemical attractants (e.g., glucose) and repellents (e.g., phenol) by swimming toward or away from them, respectively. This response is mediated by an array of receptor proteins which, upon binding a ligand, activate genes that code for proteins which, in their turn, activate the flagellar motor. The systems are adaptive - after prolonged stimulation they cease to respond, like primary neural receptors of higher organisms.

Besides this typical chemotaxis, bacteria display aerotaxis, thermotaxis, etc.

\subsubsection{Extrinsic chemical signals in multicellular organisms}

These include above all substances perceived by the sense of smell and the sense of taste. To recognize odors animals possess special cells and cell clusters with olfactory receptors, mainly glycoproteins of relative molar mass of about $10^{5}$. Upon binding to the receptor, the odogen stimulates a special $G_{0}$-protein and alerts other G-proteins in the membrane. These can activate adenylate cyclase with the final result of opening ion channels in the membrane and dispatching an impulse to the central nervous system. Humans can distinguish between 2000 and 4000 odors, the odogens being relatively small molecules of 4-20 atoms.

Pheromones of insects and some other animals belong to this group.

Tastes, on the other hand, are much less numerous, there being taste buds of only four varieties in mammals: sweet, sour, bitter and salty. Opening of $\mathrm{Na}^{+}$channels is the consequence of binding any of these 'tasty' molecules or ions. It should be observed that the many flavors we recognize in food are in fact olfactory in character.

\subsubsection{Intrinsic chemical signals in multicellular organisms}

Two classes, with some overlap, of such signals can be recognized, the hormones and the neurotransmitters.

The hormones are of several hierarchical orders as follows from the example of growth hormones. A signal arriving to the brain stimulates the hypothalamus to produce the so-called growth-hormone-releasing factor (a 44-amino-acid peptide) which stimulates the anterior pituitary to produce somatotropin (a 19-amino-acid peptide) which, in its turn, stimulates the liver to produce somatomedins (insulin-like peptides). It is only these hormones that exert their influence at target cell membranes, stimulating growth of bone and cartilage.

The membranotropic hormones, like odoriferous substances, are known to activate (or, exceptionally, inhibit) one or another of the family of $G$-proteins, of which $G_{s}, G_{i}, G_{t}, G_{0}$ with different $\alpha$, $\beta$, and $\gamma$ subunits are known. These G-proteins interact with effector molecules in or at the membrane to produce the so-called second messengers, especially cAMP.

The neurotransmitters are substances released at the presynaptic nerve endings (usually by exocytosis) that travel across the synaptic cleft to receptors at the postsynaptic nerve or muscle cell membranes. Many of them are peptides (e.g., endorphins, substance P), others are amino acids or their derivatives (e.g., glutamate, glycine, histamine, taurine) and others still catecholamines (e.g., epinephrine) and acetylcholine. In some, but not all, neurotransmitter activities, G-proteins are again involved. 


\subsubsection{The immune system}

This is a particularly complex array of cell types and interactions, developed fully only in vertebrates and especially in mammals. Macrophages, $B$ lymphocytes, $T$ lymphocytes of three types, plus a number of intercellular messengers of interleukin type, and parts of the complement system, recognize foreign antigens and produce antibodies against them, as well as convert some lymphocytes into special killer cells to annihilate the antigen.

The whole interplay is an exquisite example of membrane-exposed macromolecules mediating flow of information into channels that may be different in their mechanism of action but similar in their purpose: to maintain the organism free of adverse foreign influences.

\section{REFERENCES}

This being an overview of the present-day state of knowledge of a large part of cell biology, biochemistry and biophysics, it is not feasible to provide even a selected list of references.

A more detailed treatment of the topics covered here can be found, for instance, in

B. Alberts, D. Bray, J. Lewis, M. Raff, K. Roberts and J.D. Watson, "Molecular Biology of the Cell", second edition. Garland Publishing, Inc., New York and London, 1989.

J. Darne11, H. Lodish and D. Baltimore, "Molecular Cell Biology", second edition. Scientific American Books, New York, 1990.

A. Kotyk, K. Janáček and J. Koryta, "Biophysical Chemistry of Membrane Functions". John Wiley and Sons, Chichester, 1988.

D. Voet and J.G. Voet, "Biochemistry". John Wiley and Sons, New York, 1990.

Many relevant mini-reviews have been published over the past few years in Annual Reviews of Biochemistry, Annual Reviews of Bioenergetics, Quarterly Reviews of Biophysics and in Trends in Biochemical Sciences.

Readers who are interested in detailed references to specific topics covered here are invited to write or fax (422-4712253) to the author. 